\title{
SIZE DISTRIBUTION OF EMBRYOS PRODUCED BY CRYSTAL-ROD CONTACTS
}

\author{
A.E.D.M. VAN DER HEIJDEN \\ Research Institute of Materials, Laboratory of Solid State Chemistry, University of Nijmegen, Toernooiveld, 6525 ED Nijmegen, \\ The Netherlands \\ M. ELWENSPOEK \\ Department of Electrical Engineering, University of Twente, P.O. Box 217, 7500 AE Enschede, The Netherlands
}

and

\section{J.P. VAN DER EERDEN}

Research Institute of Materials, Laboratory of Solid State Chemistry, University of Nijmegen. Toernooiveld, 6525 ED Nijmegen, The Netherlands

Received 17 April 1989; manuscript received in final form 19 July 1989

\begin{abstract}
We performed contact nucleation experiments on the (010) face of potassium hydrogen phthalate (KAP) crystals growing in a stagnant supersaturated aqueous solution and determined - after a given growth time $t$ - (ex situ) the crystal size distribution (CSD) of the secondary nuclei (which at $t=0$ are called "embryos") by using a scanning electron microscope (SEM). The origin of the secondary nuclei could clearly be revealed (damage to the crystal surface). The CSD can be fitted with a log-normal distribution which is typical for many powders obtained by grinding. Minimum size and mean size can be quantitatively understood by elementary fracture mechanics.
\end{abstract}

\section{Introduction}

Knowledge of the crystal size distribution (CSD) is of major importance in industrial crystallization in order to control the production of new crystals. In a CMSMPR crystallizer a number of interactions between crystals or crystal-crystallizer parts are active which, each in its own way, influences the CSD: secondary nucleation, attrition, breakage and agglomeration. Further, often the smallest crystals (the fines) are removed from the crystallizer. In order to run a crystallizer at high supersaturations and to produce large crystals at high production rates, nuclei should be selectively removed from the process, so these excessive nuclei do not compete for supersaturation. Withdrawal of crystals in the larger size range is carried out to obtain a product classification. A general population equation can be derived [1] which describes how the number density $n$ (i.e. the distribution of the total number of particles over the crystal size $L$ ) varies in time taking into account the interactions and process-controlling parameters mentioned above. To solve this integro-differential equation analytically, some approximations have to be made. Firstly, one considers a clear liquid feed, i.e. the slurry which enters the crystallizer contains no crystals. Secondly, the rate processes secondary nucleation, attrition, breakage, agglomeration, fines removal and product classification can be ignored if these processes can be suppressed or if they are operative at such a minor level, that the influence on the CSD is negligible. Thirdly, one assumes that all the crystals grow with the same rate $G$ (this is generally referred to as the $\Delta L$ law of McCabe). And, finally, one assumes that the number density does not change in time, so a steady state has been

0022-0248/89/\$03.50 Elsevier Science Publishers B.V.

(North-Holland Physics Publishing Division) 
reached. This leads to the very simple, linear differential equation

$G \delta n / \delta L=-n / \tau$,

which can be solved easily to give the well-known steady-state number density

$n(L)=n_{0} \exp (-L / G \tau)$,

where $n_{0}$ is the number density at zero size, $L$ the size of a crystal and $\tau$ the residence time of the crystals in the crystallizer. It should be realized that the resulting size distribution is completely determined by the growth of crystals already present in the crystallizer and if nuclei are formed by other mechanisms, e.g. secondary nucleation, then only from size zero.

From eq. (2) it follows that if $\ln n$ is plotted versus $L$, a straight line appears with slope $-(G \tau)^{-1}$. From this slope the growth rate of the crystals can be calculated if the residence time $\tau$ is known. Numerous experiments confirm this relationship between number density and length [2-5]. However, in the lower size range always considerable deviations from the straight line are observed: the number density increases more strongly going down to smaller sizes. This effect is ascribed to birth of nuclei at finite size due to secondary nucleation and in a less degree of importance to size-dependent growth of the nuclei and growth rate dispersion. The latter means the observation that nuclei of the same size can display different growth rates. A wide variety of models for growth rate as function of size have been proposed to obtain empirical fits to the measurements (see ref. [6] for a review). Secondary nucleation at sizes larger than zero is considered as the most important source of new nuclei in an industrial crystallizer, but still little is known about its mechanism. To explain the breakdown of the linear relationship between $\ln n$ and $L$, given by eq. (2), at sizes below 10-20 $\mu \mathrm{m}$, knowledge of birth of nuclei at finite size and its size distribution is very important but still lacking.

One of the experiments to investigate secondary nucleation were carried out by Clontz and McCabe [7], who performed crystal-rod contact experiments: nuclei were produced if a rod was dropped onto a crystal in a supersaturated solution. Ex situ inspection of such crystals revealed no damage at all. Clontz and McCabe explained their results using the idea of a pre-ordered layer adjacent to the crystal surface, which was expected to contain solute clusters, microscopic dendrites and unstable agglomerates. The crystal-rod contact then would dislodge parts of the preordered liquid layer, which would either grow to nuclei of visible size or dissolve depending on whether the size of the cluster being removed was respectively larger or smaller than the critical nucleus size. Since the size of a critical nucleus is inversely proportional to the relative supersaturation, less clusters should dissolve and hence more secondary nuclei should be observed if a contact is performed in a more supersaturated solution (this so-called survival theory was introduced by Lal et al. [8]). Another explanation, and perhaps a more reasonable one according to Clontz and McCabe, was that the thickness of the pre-ordered layer increases if supersaturation is increased, and hence more clusters are available to be removed by one contact. An assumption of this model is that the size distribution of the surviving nuclei and the number of clusters to be dislodged in the first place, is independent of the supersaturation.

Light scattering experiments [9-12] revealed strange structures near a growing crystal surface, which seemed to support the idea of some preordered layer on top of the surface from which secondary nuclei could originate. One of many possible explanations (see ref. [12] for a review) was the suggestion of diffusive motion of small pre-crystalline particles formed near the solidliquid interface. However, experiments have shown that these particles have diameters larger than $\sim 300 \mathrm{~nm}$ and exhibit little or no variation in size, both of which seem highly improbable for precrystalline particles. Cummins et al. [13] proposed the so-called microbubbles hypothesis to explain the anomalous dynamic light scattering observed at the growing crystal-melt interface. They assumed a background concentration of gas present in the melt. Since the solubility of gas in a solid is smaller (by a factor 10 to 100 ) than the solubility of gas in a melt, gas segregation will take place at the interface if crystal growth is initiated. A 
quantitative confirmation of this microbubbles hypothesis was e.g. given by Livescu et al. [14], thus leaving little substantial support for the preordered layer model. Direct consequence of the proposed model by Cummins et al. [13] is that the mechanism of contact nucleation has to be sought in surface damaging rather than (the speculative) dislodging of clusters from a pre-ordered layer present near the crystal surface [7,15-17].

Experiments which subscribe the idea of surface damaging, were performed by Garside and Larson [18], who used a contacting device which merely softly touched or scraped a crystal leading to new nuclei. In contrast with ref. [7], Garside and Larson were able to observe directly secondary nucleation using a microscope, although they could not reveal details like steps or growth spirals on the surface of the mother crystal. However, it was clear that the crystal surface was damaged due to contacting. They also observed nuclei of size 20 $\mu \mathrm{m}$ and more immediately after a contact, contrasting the model proposed by Clontz and McCabe, which states that immediately after a contact only clusters of the size of a critical nucleus are present.

The CSD of secondary nuclei produced by contact nucleation has been studied by Garside et al. [19]. They performed five crystal-rod contacts in order to produce enough nuclei and to get reproducible results. The CSD was determined by counting and measuring nuclei (using a Coulter counter) within the first minute after the last contact. In this way the initial size distribution was obtained. Garside et al. interpreted their results in the same way as is usual in industrial crystallization, so plotting $\ln n$ versus $L$. For nuclei of sizes larger than about $8 \mu \mathrm{m}$ the data could be fitted using this steady-state solution which was discussed above. For sizes smaller than $8 \mu \mathrm{m}$, the data were found to deviate strongly from the straight line.

The experiments by Wissing et al. [20] used an advanced in situ microscopic technique (interference contrast) to study contact nucleation. The upper (100) surface of a potassium dihydrogen phosphate (KDP) crystal was observed from beneath the growth cell. Growth phenomena such as growth hillocks and macrosteps could easily be observed. The most important result of their work was the report of a critical impact energy $E_{\mathrm{c}}$ : below $E_{c}$ only a few $(10-100)$ secondary nuclei were produced and the mother crystal was hardly damaged, whereas above $E_{\mathrm{c}}$ a much larger amount $\left(\sim 10^{3}\right)$ of secondary nuclei was produced and, due to the contact, a small hole was formed in the crystal surface which, after some time, filled up again with crystalline material. The size of the nuclei several seconds after the impact covered a range up to $20 \mu \mathrm{m}$. A similar size range was reported by Garside and Larson [18]. Both the experiments of Wissing et al. and Garside and Larson demonstrate that due to contacting some crystalline material is chipped from the mother crystal. Furthermore, Wissing et al. found that the physical state of the surface determines the value of $E_{\mathrm{c}}$ : a surface with a high macrostep density is more easily damaged than a flat surface, hence the critical energy in the latter case will be higher than in the former (about a factor 2). This result could explain the dependence of the secondary nucleation rate on supersaturation, equilibrium temperature and impurity concentration [20]. Tai et al. [21] earlier reported a threshold energy for potassium sulphate. Below this threshold energy no nuclei were produced. Their general conclusion was that crystal growth and contact nucleation are related, with more rapidly growing crystals giving higher yields of nuclei.

The work presented here, is concerned with contact nucleation experiments on the (010) face of potassium hydrogen phthalate (KAP) crystals during growth. By combining an advanced in situ microscopic technique with an ex situ scanning electron microscopic (SEM) study of the crystalrod contact site on the crystal surface, we were able to confirm earlier observations $[18,20]$ that the secondary nuclei produced by a crystal-rod contact are formed due to attrition and hence damage of the surface. Furthermore, the SEM observations made it possible to study the CSD of the initial nuclei (or embryos).

The out-line of this paper is as follows. Section 2 deals with the details of the experimental set-up and procedure of the experiments. After that, typical crystal size distributions of secondary nuclei produced after one contact are presented as well 
as other features resulting from our experiments. In section 4 we interpret our data and other features characteristic of secondary nucleation, by introducing a simple model based on contact and fracture mechanics. After that, we want to comment on the results obtained by Garside et al. [19], which are typical examples of earlier work in this field. Their results are re-interpreted, using general results of our experiments.

\section{Experimental details}

The contacting device we used was described previously [22]. The design is such that a PVC contacting rod (having a flat tip of $0.5 \mathrm{~mm}$ diameter) hits the crystal only once; after the contact the rod is moved upwards again. The crystal surface is observed from beneath the growth cell through an optical window (on which the crystal is fixed mechanically) and the crystal itself. A double walled cell, through which thermostatically controlled water flows, was used to keep the temperature of the aqueous solution constant within $0.1^{\circ} \mathrm{C}$. The undercooling of the solution (saturated at $30.0^{\circ} \mathrm{C}$ ) was usually $2.0^{\circ} \mathrm{C}$, corresponding to a relative supersaturation of $\sigma=4.5 \%$. The temperature was measured using a thermocouple.

The microscope we used was an optical reflection microscope (interference contrast, Olympus BHM) using a $5 \times$ objective with a numerical aperture of 0.13 . The resolution of this microscope is, under optimal conditions, approximately $10 \mathrm{~nm}$ vertical and $4 \mu \mathrm{m}$ lateral.

The KAP crystals were grown from highly supersaturated $(\sigma \approx 31 \%)$ aqueous solutions of KAP (Merck, Pro Analysi, 99.8\%) using demineralized water. Because of the design of the set-up we were forced to look through the crystal at the upper surface. In order to reduce spherical aberration caused by the window and the crystal [23], the platelet-like KAP crystals should not be too thick. On the other hand, a thin crystal tends to break much easier if it is contacted with the rod. To meet both conditions, a thickness of 1.5 to $2.0 \mathrm{~mm}$ turned out to work quite satisfactorily.

We followed the next procedure during all the experiments (similar to the procedure described in ref. [22]): a KAP solution less than half a day old and saturated at $30.0^{\circ} \mathrm{C}$, was raised in temperature to $35.0^{\circ} \mathrm{C}$. After about 10 to $15 \mathrm{~min}$, the temperature was decreased to $28.0^{\circ} \mathrm{C}$. Then a crystal was cleaved (the (010) face of KAP is a cleavage plane) on both sides to provide almost atomically flat (010) surfaces. To avoid ruining of these faces, the crystal is not etched first (which will lead to the formation of many etch pits) but put in an already supersaturated solution on the observation window and fixed mechanically. After a 10 min growth period, a contact was performed. The crystal was then removed from its supersaturated solution after a certain time (the growth time). In order to reduce the supersaturated waterfilm adhering the crystal surface, it is moved through a 5-10 $\mathrm{mm}$-hexane layer which floated on top of the KAP solution. This procedure with $\mathrm{n}$-hexane is needed to reduce surface artefacts due to a sudden increase in growth rate [24].

For cleaning of the various parts of the contacting device we always used demineralized water, because normal tap-water contains a relatively high $\mathrm{Fe}^{3+}$ concentration, which is known to disturb crystal growth processes $[25,26]$.

Ex situ, the crystals are observed from above in reflected light. This clearly shows that in situ microscopy suffers an extreme loss of contrast. Furthermore, the strong spherical aberration caused by the observation window and the crystal itself in the in situ set-up [23], makes objectives with relatively higher numerical apertures $(>0.25)$, useless. Since the lateral resolution is approximately given by $\lambda / \mathrm{NA}$ (where $\lambda$ is the wavelength of the light used, $\sim 500 \mathrm{~nm}$, and NA the numerical aperture of the objective) it is seen that this quotient will be larger than $-2 \mu \mathrm{m}$. These restrictions make it impossible to obtain a clear view of the contacted site without the use of corrected lenses.

Although in situ microscopy is a very strong technique to learn more about crystal growth processes, the advantages of a scanning electron microscope with respect to optical microscopy in our specific case, are obvious: it makes oblique views and higher magnifications possible. The lateral resolution of the SEM we used (type JEOL JSM-T300) is $\sim 5 \mathrm{~nm}$. However, using a SEM 
implies the use of electrically conductive samples. Because KAP crystals are insulators, the crystal surface had to be coated with a layer of gold in a low-pressure ( $\sim 0.1$ Torr) argon atmosphere. The thickness of the gold layer is a few nm. Finally the sample is transported to the SEM to investigate the impact site.

\section{Results}

During the experiments we varied the following parameters: the height of fall of the rod (and accordingly the impact energy) and the growth time.

After a crystal was removed from the solution, the impact site was observed ex situ (from above) with the Olympus BH microscope in reflected light. Comparison of microtopographs of the impact site before and after the crystal had been coated with gold, learned that this preparation did neither ruin the characteristic surface features of KAP nor the impact site.
With the SEM, photographic close-ups were made of $60 \%$ to $85 \%$ of the contacted site. A typical example of such a close-up is shown in fig. 1. These kinds of observations clearly demonstrated that secondary nuclei are produced due to a crystal-rod contact. The nuclei do not suspend in the solution, but remain concentrated at the impact site. This explains why Derks et al. [22] did not find secondary nuclei in their experiments, besides the nuclei due to initial breeding.

As can be seen from figs. 1 and 2, the nuclei are randomly distributed over the impact site both in size and in crystallographic orientation with respect to the underlying crystal surface. Also, the nuclei are faceted and show the normal morphology of a full-grown KAP crystal. The crystal was removed from the solution as soon as possible after the crystal-rod contact and the growth time of the nuclei in fig. 1 was $\leq 10 \mathrm{~s}$. Apparently, the nuclei become faceted at least within $10 \mathrm{~s}$ after their formation.

The total area over which the secondary nuclei extend, varied from experiment to experiment, but

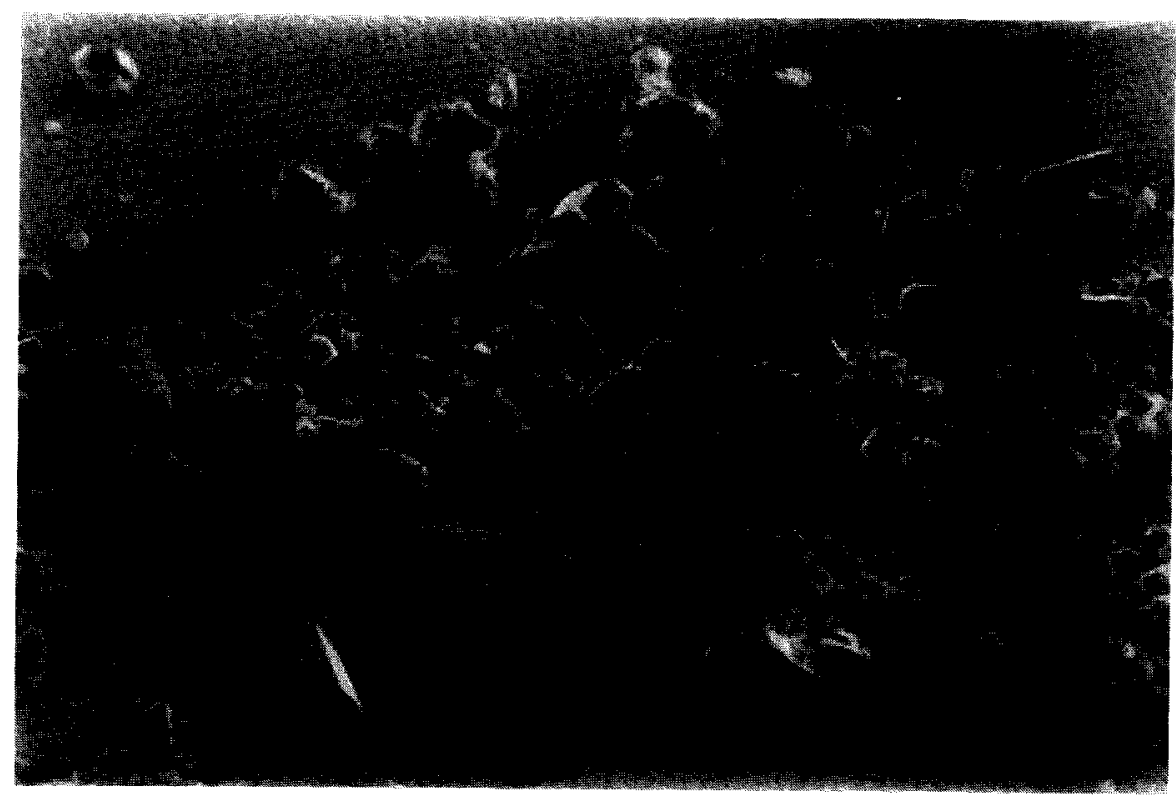

Fig. 1. SEM photograph of a part of an impact site on the (010) face of KAP (impact energy $0.35 \mathrm{~mJ}$, growth time $\leq 10 \mathrm{~s}$ ). The picture clearly shows that secondary nuclei are produced due to a crystal-rod contact; note that the nuclei are faceted. The black thin bar represents $10 \mu \mathrm{m}$ 


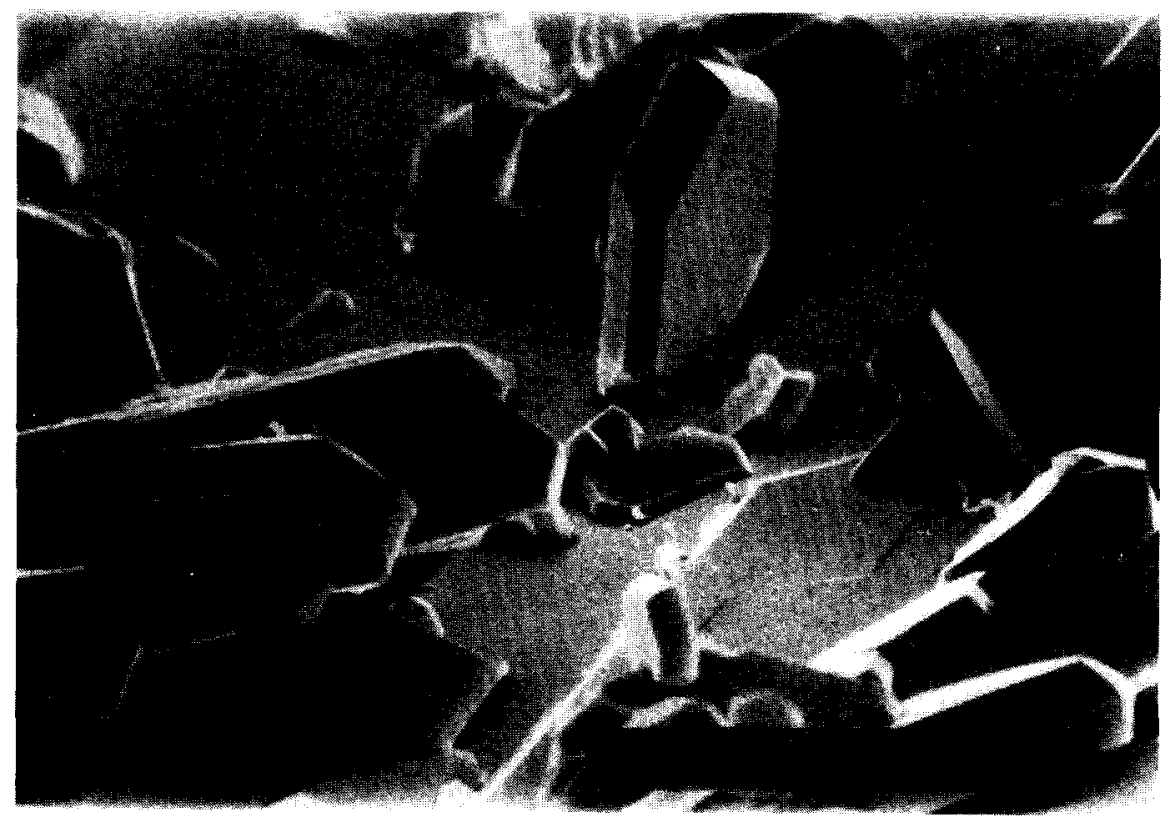

Fig. 2. SEM photograph of an impact site $(0.17 \mathrm{~mJ}, 60 \mathrm{~s})$ showing that the crystallographic orientation of the nuclei is random with respect to the orientation of the underlying mother crystal. The black thin bar represents $10 \mu \mathrm{m}$.
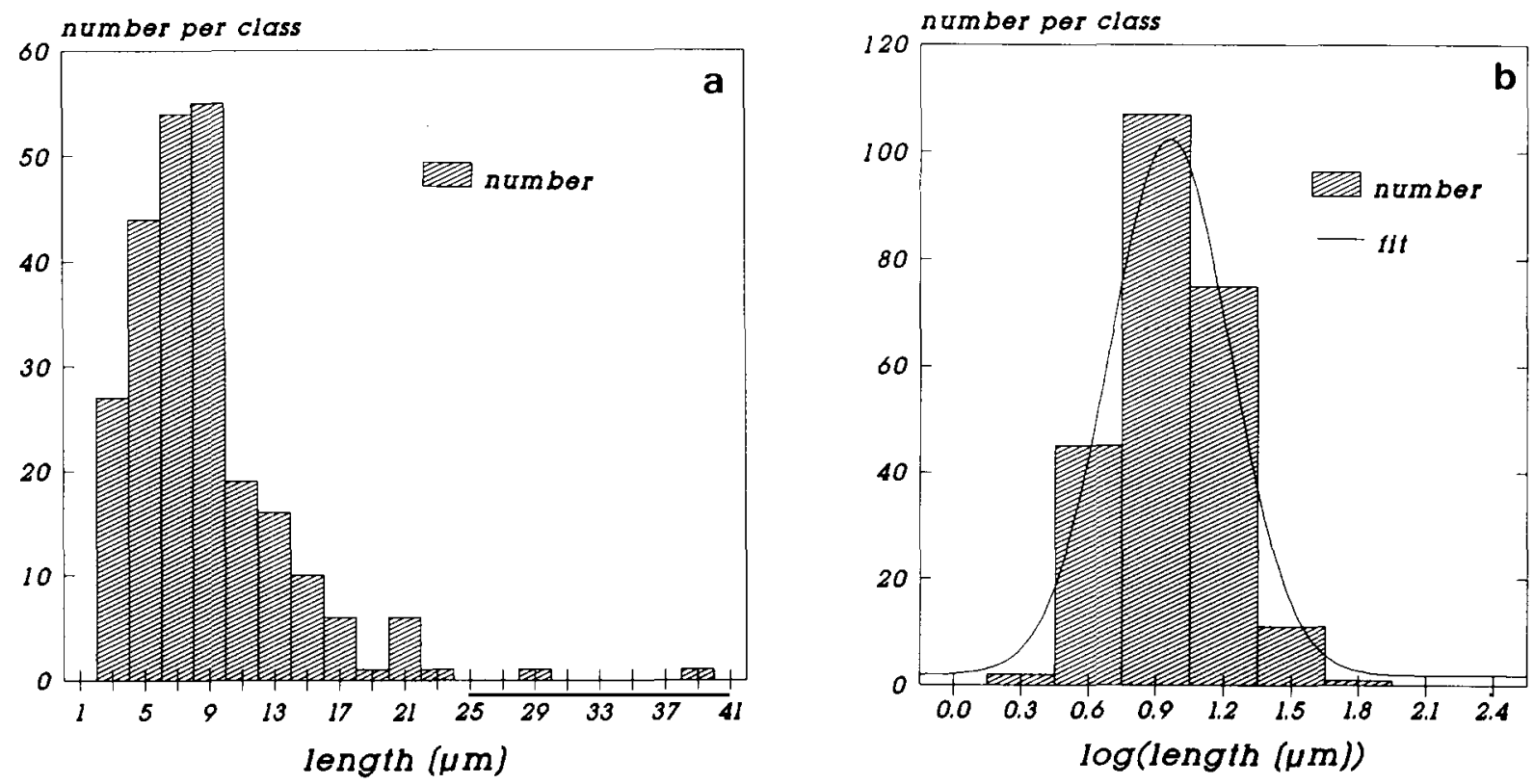

Fig. 3. (a) Typical example of a CSD of secondary nuclei with the length scale plotted linearly $(0.17 \mathrm{~mJ}, \leq 10 \mathrm{~s})$. (b) CSD which used the same data as in (a), but now the length scale is plotted logarithmically. The solid line represents the fit, based on population mean, standard deviation and peak height determined from the frequency distribution. 
never exceeded $\sim 0.5 \mathrm{~mm}^{2}$, being larger than the area of the (flat) tip which is $\sim 0.2 \mathrm{~mm}^{2}$.

The total number of nuclei per impact scattered strongly. The nuclei ranged in size (or length, which we define as the largest dimension of a nucleus) from $\sim 0.5$ to $-40 \mu \mathrm{m}$. Since the lateral resolution of the SEM is $\sim 5 \mathrm{~nm}$, this means that apparently no nuclei of sizes smaller than $\sim 0.5$ $\mu \mathrm{m}$ are formed.

For each impact site that we investigated with the SEM, we combined several photographic close-ups to cover $60 \%$ to $85 \%$ of the total number of nuclei. Because the nuclei remained concentrated at the contacted site, it was in this way, using the SEM photographs, relatively easy to measure and count these nuclei. The observation that the nuclei are randomly distributed in size over the impact site, justifies the fact that, although not all secondary nuclei produced by one contact were counted, the number that has been measured, forms a representative part of the total number of nuclei. The error in the measurement of the length of the nuclei is of the order of $10 \%$. The data are displayed in histograms with interval widths of $2.0 \mu \mathrm{m}$. (It will be clear that with decreasing the interval width, more "noise" comes up in the frequency distribution.) Fig. $3 a$ is a typical example of a CSD. The characteristics of the distributions are that there is a strong increase of particles from size $\sim 0.5 \mu \mathrm{m}$ to a certain peak value, while the tail of the distribution is much longer. This asymmetrical behaviour is typical for a log-normal distribution of which the general expression is given by

$F(L)=A \exp \left\{-\frac{\left[\log L-(\log L)_{-}\right]^{2}}{2 \sigma_{g}^{2}}\right\}$

where $A$ is the peak height, $\sigma_{\mathrm{g}}$ the standard deviation and $10^{(\log L)_{g}}$ the peak position. From eq. (3) it follows that if the horizontal scale is plotted logarithmically instead of linearly, the distribution becomes normal or Gaussian. In the semi-logarithmical plot of fig. $3 \mathrm{~b}$, the same experimental data were used as in fig. 3a, but now the distribution is symmetric.

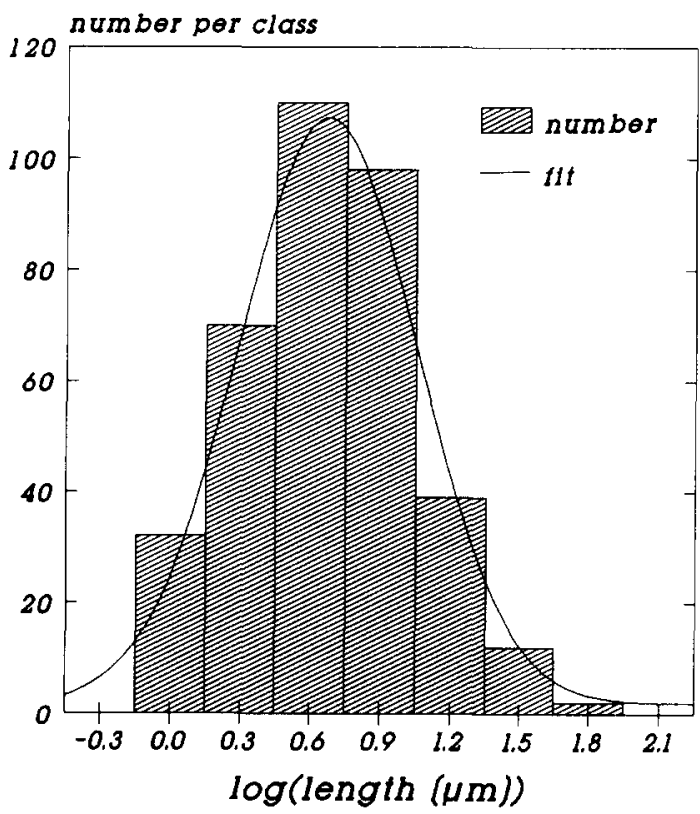

Fig. 4. CSD of secondary nuclei produced after a contact with an impact energy twice as high $(0.35 \mathrm{~mJ}, \leq 10 \mathrm{~s})$ as in previous figure; the peak has shifted to a somewhat smaller size than fig. 3.

From the histogram, the population mean defined by

$(\log L)_{\mathrm{g}} \equiv \sum_{i} \frac{f_{i} \log L_{i}}{N_{\mathrm{tot}}}$,

and the standard deviation given by

$\sigma_{\mathrm{g}}^{2} \equiv \sum_{i} \frac{f\left[\log L_{i}--(\log L)_{8}\right]^{2}}{N_{\mathrm{tot}}}$

can be calculated. In eqs. (4) and (5), $f_{i}$ is the number of nuclei within size class $\log L_{i} \pm$ $1 / 2 \log \Delta L$ and $N_{\text {tot }}$ the total number of nuclei counted. The solid line in fig. $3 \mathrm{~b}$ shows the fit (in conformity with eq. (3)) using only the parameters determined from eqs. (4) and (5) and the peak height of the frequency distribution.

We also determined crystal size distributions of secondary nuclei produced by a twice as high contact energy $(0.35 \mathrm{~mJ}$, of which an example is depicted in fig. 4) as the CSD in fig. $3(0.17 \mathrm{~mJ})$. Further, we varied the growth time of the nuclei ( $\leq 10 \mathrm{~s}, 60 \mathrm{~s}$ or $600 \mathrm{~s}$ ). Fig. 5 shows the CSD of 


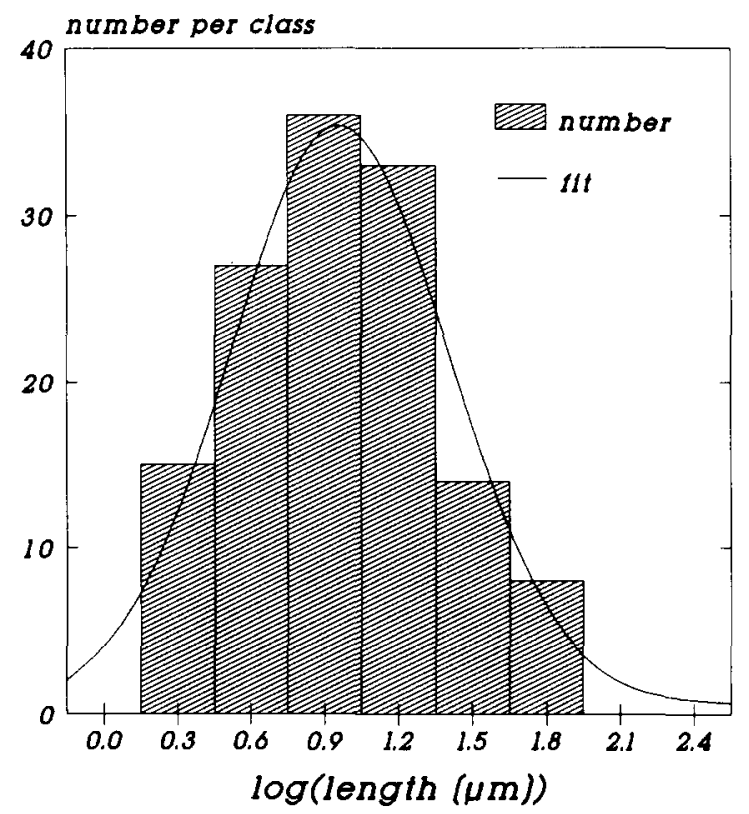

Fig. 5. CSD of secondary nuclei which were allowed to grow for $600 \mathrm{~s}(0.35 \mathrm{~mJ})$. The width of the peak has increased compared to fig. 4 .

nuclei which have grown for $600 \mathrm{~s}$ after the contact. In all these experiments, the general characteristics of the distributions are similar but shifts in peak position and peak width are observed. Table 1 gives the experimental details and fit parameters of the six distributions that were determined.

Table 1

Experimental details and fit parameters of the six crystal size distributions that were determined

\begin{tabular}{|c|c|c|c|c|c|c|}
\hline CSD-ID & $\begin{array}{l}\text { Impact } \\
\text { energy } \\
(\mathrm{mJ})\end{array}$ & $\begin{array}{l}\text { Growth } \\
\text { time } \\
\text { (s) }\end{array}$ & $(\log L)_{\mathrm{g}}$ & $\begin{array}{l}\text { Mean } \\
\text { size } \\
(\mu \mathrm{m})\end{array}$ & $\sigma_{\mathrm{g}}$ & $\begin{array}{l}\text { Peak } \\
\text { height } \\
(\#)\end{array}$ \\
\hline I & 0.17 & $\leq 10$ & 0.96 & 9.1 & 0.25 & 108 \\
\hline II & 0.35 & $\leq 10$ & 0.66 & 4.6 & 0.31 & 221 \\
\hline III & 0.35 & $\leq 10$ & 0.67 & 4.7 & 0.37 & 110 \\
\hline IV & 0.17 & 60 & 1.01 & 10.2 & 0.26 & 163 \\
\hline V & 0.35 & 60 & 0.90 & 7.9 & 0.25 & 206 \\
\hline VI & 0.35 & 600 & 0.96 & 9.1 & 0.44 & 36 \\
\hline
\end{tabular}

\section{Discussion}

First we want to make a general remark on a log-normal distribution: it is encountered in several physical (but also in e.g. economical) problems. For instance, the sizes of particles in a powder, formed by grinding, are often log-normally distributed. What we want to indicate is, that a log-normal distribution is not just a mathematical function which is capable of fitting some specific data satisfactorily, but that it may have a physical background as well. For example the physics behind the log-normal size distribution of particles in a powder is that the $i$ th grinding process forms fractions of particles which were formed during the $(i-1)$ th grinding. So if one starts of with a particle of size $X$, the first grinding will produce a particle of size $\xi_{1} X$; the second one of size $\xi_{2}\left(\xi_{1} X\right)$, etc. If the grinding proportions $\xi_{i}$ are independent of the size of the particle and the index $i$, then the repeated grinding will eventually lead to a log-normal distribution of the size of the particles in the powder.

The error of $-10 \%$ in the length $L$ of the nuclei stems from the fact that the largest dimension of a nucleus is, in general, not parallel to the plane of the SEM photographs, being oblique views as well. However, here another, almost classical problem raises its head: attributing a size to an arbitrarily shaped particle which best describes reality. For instance, a Coulter counter (which is often used to determine size distributions, see e.g. refs. [3,19]) measures volumes of particles passing a small orifice and then calculates effective radii on the basis of spherical particles. But if the particles measured are cubes or cylinders, the error in size (if no correction is applied) will also be in the $10-20 \%$ range. This implies that size distributions, especially of nonspherical particles and regardless of how they are determined, have to be interpreted with great care.

The crystal size distributions plotted semi-logarithmically, can be fitted very well with a log-normal distribution, given by eq. (3). The fit uses peak height and the statistical parameters population mean, eq. (4), and standard deviation, eq. (5), which all follow from the frequency distributions.

In order to make a statement on the acceptabil- 
ity of the proposed log-normal fit to the experimental data, a Kolmogorov-Smirnov test was carried out. We will not go into details of this test, but refer to one of many textbooks on probability and statistics in which this kind of hypothesis testing is described [27]. The results of this test were that all the populations satisfy the proposed log-normal distribution up to $99 \%$ reliability, which is very good according to statistical standards [27].

Although we are not able to make unambiguous, quantitative statements on a (possible) relation between impact energy and growth time on one hand and peak position, peak width and total number of nuclei on the other, due to the limited number of experiments, it seems that a higher impact energy produces somewhat smaller nuclei (see table 1); however, this could be compensated by a broader (smaller) distribution or different peak height, such that in either case e.g. the total volume of all the nuclei (approximately) remains the same. Further, the population means of distributions of nuclei with longer growth times are shifted to somewhat larger values, implying growth of the nuclei (compare the series II, III, V and VI of table 1). On the other hand, taking into account the error in determining the sizes of the secondary nuclei, the shifts in these values are probably marginal.

The fact that also the distributions of secondary nuclei with longer growth times can be described with a log-normal distribution (see fig. 5 ), suggests that the nuclei either grow all with the same rate or grow very slowly, not necessarily with the same rate. Many papers on secondary nucleation using different crystalline materials report a very wide growth rate dispersion of secondary nuclei, often size-dependent; even nuclei which displayed no growth at all have been observed $[18,28-30]$. Hence, the statement that all the nuclei in one experiment grow with the same rate in this way maintaining a log-normal distribution in time, will be very unlikely. The reason for the growth rate dispersion is probably the assessment of stress in a nucleus, which in general will vary from one to another. This has been demonstrated by Ristic et al. [31], who found a direct correlation between stress density and growth rate of secondary nuclei: the more strain, the lower the growth rate.
In spite of the low growth rate, the nuclei are faceted (see figs. 1 and 2). From elementary crystal growth theory [32] it follows that the radius $R$ of a sphere surrounded by a mother phase changes in time $t$ proportional to $\sqrt{D t}$, with $D$ the diffusion constant (if the growth rate is diffusion controlled). It is very likely to assume that the fragments chipped from the surface are not faceted; they are probably not spherical either, but it is the most simple assumption that can be made at this stage. Hence, we can make a rough estimate of the time needed to transfer a spherical particle into a faceted one. This transfer time will be of the order of $R^{2} / D$, which takes a value of $10-100 \mathrm{~ms}$ for a $10 \mu \mathrm{m}$ sphere radius and a diffusion constant which for aqueous solutions is typically $10^{-9} \mathrm{~m}^{2} / \mathrm{s}$. So, faceting of the secondary nuclei occurs virtually immediately after their formation. We can conclude from this rather short transfer time that the growth rate involved in the faceting process, is considerably larger than the growth rates of the nuclei that have completed this process. To explain this, it should be reminded that the fastes growing faces that establish the faceting are rough (no crystallographic direction) and should grow with maximum growth rate, which is diffusion controlled, while the remaining faces are the ones with the lowest growth rates.

The following step in this discussion is the introduction of some contact and fracture mechanical concepts, in trying to explain the minimum size of the secondary nuclei $(-0.5 \mu \mathrm{m})$ and the small spread in mean size of the crystal size distributions if different impact energies are used. We do this by analyzing, step by step, the movement of the rod on its way down to the crystal and the actual contact.

When the rod falls from a defined height, it experiences a frictional force due to the viscosity $\eta$ of the medium through which the rod moves and buoyancy on the part of the rod which is submerged in the solution. However, the viscosity and density in the case of a KAP solution as well as the velocity of the rod, are such, that these forces are negligible compared to gravity. During the fall, in front of the rod, fluid has to be pushed away. In ref. [33] we show that this resistant force becomes significant if the distance between the 


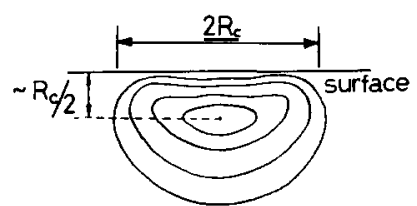

Fig. 6. Stress concentration profile in a material due to loading with a spherical indenter; $R_{\mathrm{c}}$ is the radius of contact. Point of maximum stress is located at a distance $\sim R_{\mathrm{c}} / 2$ below the surface (after Davies [34]).

rod and the crystal surface is of the order of 100 $\mu \mathrm{m}$. The energy lost to push away the remaining fluid between rod and surface has been estimated at $1-5 \%$. From this we can conclude that the potential energy of the rod before the contact is a reasonable measure of the energy transferred to the crystal.

During the loading of the rod on the crystal surface, the propagation of the elastic deformation will be almost instantaneous, because the velocity of sound in the crystal is $-10^{3}-10^{4}$ times larger than the penetration velocity of the rod in the surface. More and more stress is applied and at a certain time dislocation loops or even cracks will be formed. Since the highest concentration of stress during a contact is at a point in the crystal at a distance from the surface approximately equal to half of the radius of projection of the contact area on the surface (see fig. 6 after Davies [34]), these cracks will nucleate $\sim 100-150 \mu \mathrm{m}$ below the surface (in fact these subsurface cracks have been observed during similar experiments in our laboratory, see ref. $[22,35])$. At this moment, the plane containing the subsurface cracks (labelled $\mathrm{C}$ in fig. 7) acts as a boundary layer through which the propagation of the elastic deformation will be much more difficult. If loading still continues,

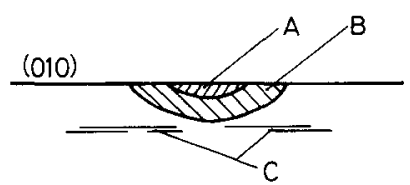

Fig. 7. Schematic picture of the contact site at and some hundred $\mu \mathrm{m}$ below the crystal surface. Region A: region of severe plastic deformation, which finally fragmentizes; region B: elastically deformed region bounded by subsurface cracks, labelled $\mathrm{C}$. then the remaining energy will be absorbed by the bounded region (labelled B) and part of this region (labelled A) will suffer severe (plastic) deformation. In the deformed region the stress density will increase and if a certain yield stress is exceeded, eventually lead to the formation of new surface area: a part of the bounded region breaks apart into fragments, which in general will contain a certain amount of stress. From fracture mechanics it is known [36] that fragments smaller than a certain size, say $l_{0}$, will not be formed. This size $l_{0}$, given by

$l_{0}=\alpha E \Gamma / Y^{2}$,

separates brittle $\left(L>l_{0}\right)$ from ductile $\left(L<l_{0}\right)$ fragments, and is determined by three material constants, i.e. Young's modulus $E$, the fracture surface energy $\Gamma$ and the uniaxial yield stress $Y$, and the constant $\alpha$, characteristic of the performed test. Substituting some typical material constants: $E \sim 10^{8} \mathrm{~N} / \mathrm{m}^{2}, \Gamma \sim 1 \mathrm{~J} / \mathrm{m}^{2}, Y \sim 10^{7}$ $\mathrm{N} / \mathrm{m}^{2}$ and $\alpha \sim 1$ into eq. (6), gives a ductile/brittle size of $\sim 1 \mu \mathrm{m}$, which agrees with the observed minimum size of $-0.5 \mu \mathrm{m}$ of secondary nuclei. It is known [37] that fragments of size $l_{0}$ and smaller can sustain relatively high strains and hence are able to contain very high stress densities (and yet do not crack), which evidently could lead to very low growth rates of these small nuclei, in accordance with ref. [31].

Let us assume that $N$ spheres of radius $r$ with total surface area $A$ are formed out of a volume $V$, then from energy conservation it follows that

$V\left(Y_{\text {dyn }}-Y_{\text {stat }}\right)=A \Gamma$,

where $Y_{\text {dyn }}$ and $Y_{\text {stat }}$ are respectively the dynamical and static yield stress. Since in this model $V=$ $4 \pi N r^{3} / 3$ and $A=4 \pi N r^{2}$, a mean size $r$ of the fragments can be calculated from eq. (7):

$r=\frac{3 \Gamma}{\left(Y_{\mathrm{dyn}}-Y_{\mathrm{stat}}\right)}$,

which results in $r \sim 6 \mu \mathrm{m}$ for $\Gamma \sim 1 \mathrm{~J} / \mathrm{m}^{2}$ and $Y_{\text {stat }} \sim 0.95 Y_{\text {dyn }} \sim 10^{7} \mathrm{~N} / \mathrm{m}^{2}$. This corresponds very well to the population means of the crystal size distributions (see table 1). 
The depth of the subsurface cracks which bounds a region, containing the volume $V$ that suffers severe deformation and finally falls apart, is directly correlated with the tip radius of the rod, whereas its impact energy merely determines the stress concentration profile in the crystal [34]. This contact mechanical point of view could explain why the population characteristics determined under different experimental conditions, do not diverge significantly.

Let us now comment on the results of Garside et al. [19] on the CSD of secondary nuclei already mentioned. In principle it is very unlikely that the CSD of secondary nuclei should show the same dependence as the steady-state solution of an industrial crystallizer, since the assumptions made to derive this solution, are not at all applicable to crystal-rod contact nucleation experiments. Still, these interpretations are commonly used in similar contact nucleation experiments as described in ref. [19]. On basis of the available results in this paper (especially the log-normal character of the CSD of secondary nuclei) we can re-interpret some of the results of ref. [19].

From fig. 5 of ref. [19],

$N_{\text {cum }}(L)=\int_{L_{0}}^{L} n\left(L^{\prime}\right) \mathrm{d} L^{\prime}$

can be calculated, where $N_{\text {cum }}(L)$ is the cumulative undersize of the distribution, i.e. the total number of particles smaller than size $L$, and $L_{0}$ the smallest size measured $(2.0 \mu \mathrm{m})$. The total number of nuclei $N_{\text {to }}$ follows by integrating eq. (9) to infinity. Dividing eq. (9) by $N_{\text {tor }}$ leads to a relative, cumulative undersize distribution, which is plotted versus $\log L$. Because we now deal with a cumulative distribution, the following integral has to be used to fit the data:

$$
(2 \pi)^{-1 / 2} \sigma_{\mathrm{g}}^{-1} \int_{-\infty}^{y} \exp \left[-\left(y^{\prime}-y_{\mathrm{g}}\right)^{2} / 2 \sigma_{\mathrm{g}}^{2}\right] \mathrm{d} y^{\prime},
$$

which can be transformed to the standard normal distribution:

$$
(2 \pi)^{-1 / 2} \int_{-\infty}^{\left(y-y_{g}\right) / \sigma_{g}} \exp \left[-x^{2} / 2\right] \mathrm{d} x,
$$

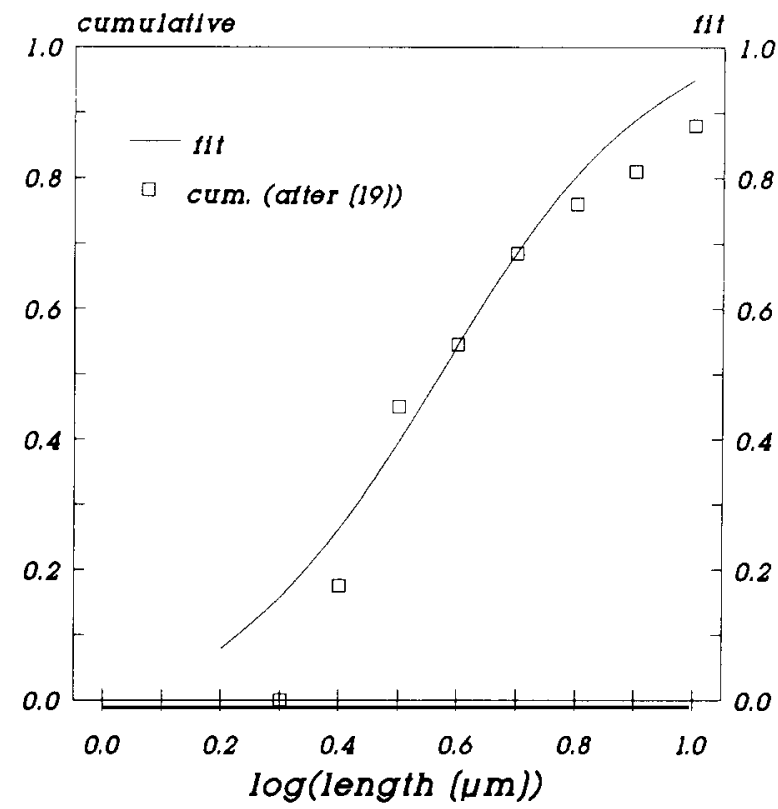

Fig. 8. Log-normal fit (solid line) of some of the data (square markers) from Garside et al. [19] (note that this is a cumulative distribution) showing reasonable agreement.

using the substitution $x=\left(y^{\prime}-y_{\mathrm{g}}\right) / \sigma_{\mathrm{g}}$. Eq. (11) is defined as the error function and is tabulated in [38]. In fig. 8, the result of this fit is shown. Although there is some misagreement in the larger size region (which may be due to the fact that five contacts have been performed in stead of one) the fit is satisfying. From this we conclude that also previous results on CSD of secondary nuclei produced by crystal-rod contacts satisfy a log-normal distribution.

Although there is an analogon between the CSD of secondary nuclei produced by a crystalrod contact and the size distribution of particles in a powder produced by grinding, both being lognormal, the physical background of these processes will be different, since the latter is based on a repeated process, while the former is just one contact. Therefore, there has to be another physical explanation for the log-normal size distribution of the embryos.

A possible entry to the physical background of the log-normal CSD might be the surface tensile stress field around the indentation in the surface made by the contacting rod. For plastic-elastic 
materials this stress field is modeled by determining the stress field around a hole of radius $a$ expanded by internal pressure in an infinite plate. The surface circumferential tensile stress as a function of the radius $r$ from the centre of the plastically deformed circular region in an isotropic medium is given by [36]:

$\sigma_{\theta}(r)=\frac{1}{2} Y(1-2 \ln c / r), \quad a<r<c$,

where $c$ is the radius of the plastic zone. Outside the plastic region $(r>c)$, the stress falls off with $1 / r^{2}$. There is no doubt that in reality the stress field is much more complicated, moreover since KAP is highly anisotropic. However it might very well be possible that the $\ln r$ dependence of the surface tensile stress is responsible for the logarithmic behaviour of the sizes in the CSDs.

\section{Conclusions}

Much work on determination of crystal size distributions of secondary nuclei produced by contact nucleation, explicitly made use of the fact that the nuclei become suspended in the solution, so they can be measured either using a Coulter counter, a sieve analysis technique, a laser-scattering based device or combinations (see, e.g., refs. $[2-5,18])$. Up to now practically all the distributions presented in literature are steady state CSDs. In this paper we studied ex situ impact sites on KAP(010) surfaces carried out during growth of the crystals, by using a scanning electron microscope (SEM). The SEM observations showed that the secondary nuclei are faceted and randomly distributed in both size and crystallographic orientation over the contacted site. Estimations show that faceting of the nuclei occurs virtually immediately $(10 \sim 100 \mathrm{~ms})$ after the contact.

The most important conclusion is that the CSDs of the secondary nuclei are log-normal, which means that if the number of nuclei in a certain size class is plotted versus the logarithm of the size of the nuclei the distribution becomes Gaussian. We are able to understand the measured parameters like minimum size and mean size of the nuclei using elementary fracture mechanics. The devia- tion from the steady state number density for a CMSMPR crystallizer when going down to smaller sizes, stems from birth of nuclei into a finite size range, which can be estimated using eqs. (6) and (8) (note that these formulas are almost completely determined by material constants).

\section{Acknowledgements}

We want to thank H.P.M. Geurts for technical assistance and P. Bennema, H. Meekes and R.C. de Boer for helpful discussions. A.E.D.M. van der Heijden wishes to acknowledge financial support from the Stichting voor de Technische Wetenschappen (STW).

\section{References}

[1] See, e.g., S.J. Jančić and P.A.M. Grootscholten, in: Industrial Crystallization '84, Eds. S.J. Jančić and E.J. de Jong (Elsevier, Amsterdam, 1984) ch. 4.

[2] J.E. Helt and M.A. Larson, AIChE J. 23 (1977) 822.

[3] J. Garside and S.J. Jančić, AIChE J. 25 (1979) 948.

[4] A.G. Jones, J. Budz and J.W. Mullin, AIChE J. 32 (1986) 2002.

[5] P.J. Daudey, Doctor's Thesis, Delft University of Technology (1987).

[6] E.T. White, L.L. Bendig and M.A. Larson, AIChE J. Symp. Ser. 72 (153) (1976) 41.

[7] N.A. Clontz and W.L. McCabe, Chem. Eng. Progr. Symp. Ser. No. 110, 67 (1971) 6.

[8] D.P. Lal, R.E.A. Mason and R.F. Strickland-Constable, J. Crystal Growth 5 (1969) 1.

[9] J.H. Bilgram, H. Güttinger and W. Känzig, Phys. Rev. Letters 40 (1978) 1394.

[10] P. Böni, J.H. Bilgram and W. Känzig, Phys. Rev. A28 (1983) 2953.

[11] O.N. Mesquita, D.G. Neal, M. Copic and H.Z. Cummins, Phys. Rev. B29 (1984) 2846.

[12] O.N. Mesquita and H.Z. Cummins, Physico-Chem. Hydrodyn. 5 (1984) 389.

[13] H.Z. Cummins, G. Livescu, H. Chou and M.R. Srinivasan, Solid State Commun. 60 (1986) 857.

[14] G. Livescu, M.R. Srinivasan, H. Chou and H.Z. Cummins, Phys. Rev. A36 (1987) 2293.

[15] M. Elwenspoek and P. Bennema in: Industrial Crystallization '84, Eds. S.J. Jančić and E.J. de Jong (Elsevier, Amsterdam, 1984) p. 267.

[16] M.A. Larson and L.L. Bendig, AIChE Symp. Ser. No. 153, 72 (1976) 12.

[17] G.A. Hussmann, M.A. Larson and K.A. Berglund, in: 
Industrial Crystallization '84, Eds. S.J. Jančić and E.J. de Jong (Elsevier, Amsterdam, 1984) p. 21.

[18] J. Garside and M.A. Larson, J. Crystal Growth 43 (1978) 694.

[19] J. Garside, I.T. Rusli and M.A. Larson, AIChE J. 25 (1979) 57.

[20] R. Wissing, M. Elwenspoek and B. Degens, J. Crystal Growth 79 (1986) 614.

[21] C.Y. Tai, W.L. McCabe and R.W. Rousseau, AIChE J. 21 (1975) 351.

[22] M.P.W. Derks, A.E.D.M. van der Heijden and $M$. Elwenspoek, J. Crystal Growth 94 (1989) 527.

[23] M. Elwenspoek, J. Crystal Growth 87 (1988) 375.

[24] W.J.P. van Enckevort, R. Janssen-van Rosmalen and W.H. van der Linden, J. Crystal Growth 49 (1980) 502.

[25] M.H.J. Hottenhuis and C.B. Lucasius, J. Crystal Growth 78 (1986) 379.

[26] M.H.J. Hottenhuis and A. Oudenampsen, J. Crystal Growth 92 (1988) 513

[27] B.W. Lindgren and G.W. McElrath, in: Introduction to Probability and Statistics (Macmillan, New York, 1959) ch. 8 .
[28] R.J. Davey, R.I. Ristić and B. Žižić, J. Crystal Growth 47 (1979) 1.

[29] J. Garside and R.I. Ristić, J. Crystal Growth 61 (1983) 215.

[30] K. Shimizu, H. Kato and N. Kubota, J. Crystal Growth 85 (1987) 543.

[31] R.J. Ristic,, J.N. Sherwood and K. Wojciechowski, J. Crystal Growth 91 (1988) 163.

[32] See, e.g. A.A. Chernov, Modern Crystallography III: Crystal Growth, Springer Series in Solid State Sciences 36 (Springer, Berlin, 1984).

[33] A.E.D.M. van der Heijden, M. Elwenspoek and J.P. van der Eerden, to be published.

[34] R.M. Davies, Proc. Roy. Soc. (London) A197 (1949) 416.

[35] A.E.D.M. van der Heijden and M. Elwenspoek, poster presented at ICCG-9, Sendai, Japan, August 1989.

[36] K.E. Puttick, J. Phys. D (Appl. Phys.) 11 (1978) 595.

[37] K.E. Puttick and A.S.T. Badrick, Chem. Eng. Sci. 42 (1987) 855 .

[38] M. Abramowitz and I.A. Stegun, Handbook of Mathematical Functions (Dover, New York, 1970). 